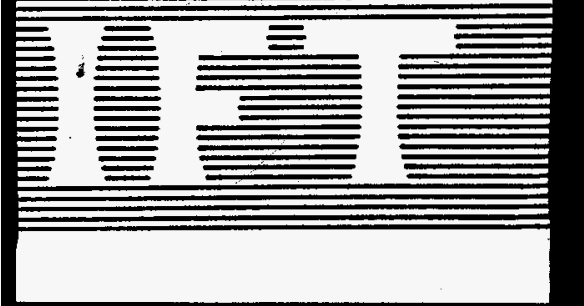

\title{
DOEERR/40272--294
}

\section{Quantum Transformations}

\author{
Alon E. Faraggi ${ }^{1}$ and Marco Matone ${ }^{2}$ \\ 1 Institute for Fundamental Theory, Department of Physics, \\ University of Florida, Gainesville, FL 32611, USA \\ e-mail: faraggi@phys.ufl.edu \\ 2 Department of Physics "G. Galilei" - Istituto Nazionale di Fisica Nucleare \\ University of Padova, Via Marzolo, 8 - 35131 Padova, Italy \\ e-mail: matone@padova.infn.it
}

We show that the quantum Hamilton-Jacobi equation can be written in the classical form with the spatial derivative $\partial_{q}$ replaced by $\partial_{\hat{q}}$ with $d \hat{q}=\frac{d q}{\sqrt{1-\beta^{2}(q)}}$, where $\beta^{2}(q)$ is strictly related to the quantum potential. This can be seen as the opposite of the problem of finding the wave function representation of classical mechanics as formulated by Schiller and Rosen. The structure of the above "quantum transformation", related to the recently formulated equivalence principle, indicates that the potential deforms space geometry. In particular, a result by Flanders implies that both $\mathcal{W}(q)=V(q)-E$ and the quantum potential $Q$ are proportional to the curvatures $k_{\mathcal{W}}$ and $k_{Q}$ which arise as natural invariants in an equivalence problem for curves in the projective line. In this formulation the Schrödinger equation takes the geometrical form $\left(\partial_{q}^{2}+k_{W}\right) \psi=0$.

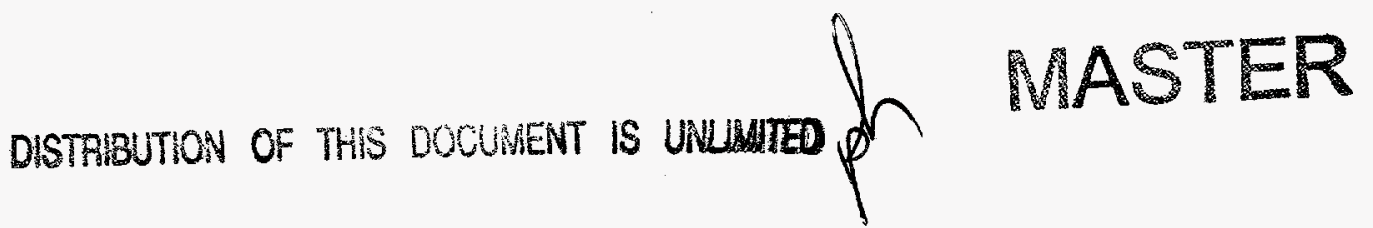


Let us denote by $\mathcal{S}_{0}$ the Hamiltonian characteristic function (also called classical reduced action), so that $p=\partial_{q} \mathcal{S}_{0}$, where $q$ and $p$ are the coordinate and the momentum respectively. The properties of $\mathcal{S}_{0}$ are fixed by the equivalence principle introduced in [1] and that will be recalled below. Let us consider the case of two physical systems with Hamilton's characteristic functions $\mathcal{S}_{0}$ and $\mathcal{S}_{0}^{v}$ and denote the coordinates of the two systems by $q$ and $q^{v}$ respectively. Setting

$$
\mathcal{S}_{0}^{v}\left(q^{v}\right)=\mathcal{S}_{0}(q),
$$

induces the " $v$-transformations"

$$
q \longrightarrow q^{v}=v(q),
$$

where $v=\mathcal{S}_{0}^{\nu-1} \circ \mathcal{S}_{0}$, with $\mathcal{S}_{0}^{\nu}$ denoting the inverse of $\mathcal{S}_{0}^{v}$.

Recently, the following problem has been considered in $[1,2]$

Given an arbitrary system with reduced action $\mathcal{S}_{0}(q)$, find the coordinate transformation $q \longrightarrow q^{v_{0}}=v_{0}(q)$, such that the new reduced action $\mathcal{S}_{0}^{v_{0}}$, defined by

$$
\mathcal{S}_{0}^{v_{0}}\left(q^{v_{0}}\right)=\mathcal{S}_{0}(q)
$$

corresponds to the free system with vanishing energy.

In the following we will use the notation $q^{0} \equiv q^{v_{0}}, \mathcal{S}_{0}^{0} \equiv \mathcal{S}_{0}^{v_{0}}$. We also set $\mathcal{W}(q) \equiv V(q)-E$, and denote the $\mathcal{W}=0$ state by

$$
\mathcal{W}^{0}\left(q^{0}\right) \equiv 0 .
$$

Observe that the structure of the states described by $\mathcal{S}_{0}^{0}$ and $\mathcal{S}_{0}$ determines the "trivializing coordinate" $q^{0}$ to be

$$
q \longrightarrow q^{0}=\mathcal{S}_{0}^{0^{-1}} \circ \mathcal{S}_{0}(q),
$$

Let us denote by $\mathcal{H}$ the space of all possible $\mathcal{W}$ 's. The above question suggested the "diffeomorphic equivalence principle" [1]

For each pair $\mathcal{W}^{a}, \mathcal{W}^{b} \in \mathcal{H}$, there is a $v$-transformation such that

$$
\mathcal{W}^{a}(q) \longrightarrow \mathcal{W}^{a v}\left(q^{v}\right)=\mathcal{W}^{b}\left(q^{v}\right)
$$

Observe that this implies that there always exists the trivializing coordinate $q^{0}$ for which $\mathcal{W} \rightarrow \mathcal{W}^{0}$ 


\section{DISCLAIMER}

This report was prepared as an account of work sponsored by an agency of the United States Government. Neither the United States Government nor any agency thereof, nor any of their employees, makes any warranty, express or implied, or assumes any legal liability or responsibility for the accuracy, completeness, or usefulness of any information, apparatus, product, or process disclosed, or represents that its use would not infringe privately owned rights. Reference herein to any specific commercial product, process, or service by trade name, trademark, manufacturer, or otherwise does not necessarily constitute or imply its endorsement, recommendation, or favoring by the United States Government or any agency thereof. The views and opinions of authors expressed herein do not necessarily state or reflect those of the United States Government or any agency thereof. 


\section{DISCLAIMER}

Portions of this document may be illegible electronic image products. Images are produced from the best available original document. 
In [1] it has been shown that this principle implies the quantum analogue of the HamiltonJacobi equation which in turns implies the Schrödinger equation. Subsequently it has been shown in [2] that this is the unique possible solution. Let us shortly review the structure of the derivation in $[1,2]$. First of all one observes the basic fact that the equivalence principle cannot be consistently implemented in classical mechanics. This can be summarized in the following steps

1) Consider the Classical Stationary Hamilton-Jacobi Equation (CSHJE): $\frac{1}{2 m}\left(\partial_{q} \mathcal{S}_{0}^{c l}\right)^{2}+$ $\mathcal{W}=0$

2) given another system with reduced action $\mathcal{S}_{0}^{c l v}$, denote by $q^{v}$ the independent space coordinate variable;

3) set $q^{v}=v(q)$ with $v$ determined by $\mathcal{S}_{0}^{c l v}\left(q^{v}\right)=\mathcal{S}_{0}^{c l}(q)$, that is $v=\mathcal{S}_{0}^{c l v}{ }^{-1} \circ \mathcal{S}_{0}^{c l}$;

4) compare the CSHJE for the $\mathcal{S}_{0}^{c l v}$ system, that is $\frac{1}{2 m}\left(\partial_{q}^{v} \mathcal{S}_{0}^{c l v}\left(q^{v}\right)\right)^{2}+\mathcal{W}^{v}\left(q^{v}\right)=0$ with $\frac{1}{2 m}\left(\partial_{q} \mathcal{S}_{0}^{c l}(q)\right)^{2}+\mathcal{W}(q)=0$ and use $\mathcal{S}_{0}^{c l v}\left(q^{v}\right)=\mathcal{S}_{0}^{c l}(q)$ so that consistency implies $\mathcal{W}^{v}\left(q^{v}\right)=\left(\partial_{q} q^{v}\right)^{-2} \mathcal{W}(q)$. Therefore, in classical mechanics $\mathcal{W}$ belongs to $\mathcal{Q}$, the space of functions transforming as quadratic differentials under $v$-maps;

5) it follows that in classical mechanics the $\mathcal{W}^{0}$ state is a fixed point in the space of possible states, namely under a coordinate transformation $\mathcal{W}^{0}\left(q^{0}\right) \longrightarrow\left(\partial_{q^{0}} q^{v}\right)^{-2} \mathcal{W}^{0}\left(q^{0}\right)=0$.

It is therefore clear that in order to implement the equivalence principle the CSHJE should be modified. The most general form would be

$$
\frac{1}{2 m}\left(\frac{\partial \mathcal{S}_{0}(q)}{\partial q}\right)^{2}+\mathcal{W}(q)+Q(q)=0
$$

Since classical mechanics exists, it is clear that the above equation must reduce to the CSHJE in a suitable limit. That is in some limit we must have

$$
Q \rightarrow 0 \text {. }
$$

Since the equivalence principle implies that $\mathcal{W} \notin \mathcal{Q}$, it is clear that classical mechanics is the covariance breaking phase with $Q$ having the role of covariantizing term.

The properties of $\mathcal{W}+Q$ under the $v$-transformations are determined by the transformed equation $\left(\partial_{q^{v}} \mathcal{S}_{0}^{v}\left(q^{v}\right)\right)^{2} / 2 m+\mathcal{W}^{v}\left(q^{v}\right)+Q^{v}\left(q^{v}\right)=0$ that by (1) and (7) yields

$$
\mathcal{W}^{v}\left(q^{v}\right)+Q^{v}\left(q^{v}\right)=\left(\partial_{q} q^{v}\right)^{-2}(\mathcal{W}(q)+Q(q))
$$


that is

$$
(\mathcal{W}+Q) \in \mathcal{Q} .
$$

Let us recall how $Q$ is determined by the equivalence principle $[1,2]$. We have seen that if $\mathcal{W}$ transforms as a quadratic differential, then $\mathcal{W}^{0}$ would be a fixed point in the $\mathcal{H}$ space. It follows that $\mathcal{W} \notin \mathcal{Q}$ so that by (10) $\mathcal{Q} \notin \mathcal{Q}$. Therefore

$$
\mathcal{W}^{v}\left(q^{v}\right)=\left(\partial_{q^{a}} q^{v}\right)^{-2} \mathcal{W}^{a}\left(q^{a}\right)+\left(q^{a} ; q^{v}\right)
$$

and by $(10)$

$$
Q^{v}\left(q^{v}\right)=\left(\partial_{q^{a}} q^{v}\right)^{-2} Q^{a}\left(q^{a}\right)-\left(q^{\alpha} ; q^{v}\right) .
$$

For $\mathcal{W}^{a}\left(q^{a}\right)=\mathcal{W}^{0}\left(q^{0}\right)$ Eq.(11) gives

$$
\mathcal{W}^{v}\left(q^{v}\right)=\left(q^{0} ; q^{v}\right)
$$

This means that all the states correspond to the inhomogeneous part of the transformation of the $\mathcal{W}^{0}$ state induced by some diffeomorphism.

Let $a, b$ and $c$ denote arbitrary $v$-transformations. Comparing

$$
\mathcal{W}^{b}\left(q^{b}\right)=\left(\partial_{q^{b}} q^{a}\right)^{2} \mathcal{W}^{a}\left(q^{a}\right)+\left(q^{a} ; q^{b}\right)=\left(q^{0} ; q^{b}\right),
$$

with the same formula with $q^{a}$ and $q^{b}$ interchanged we have $\left(q^{b} ; q^{a}\right)^{-}=-\left(\partial_{q^{a}} q^{b}\right)^{2}\left(q^{a} ; q^{b}\right)$, in particular $(q ; q)=0$. More generally, comparing

$$
\begin{gathered}
\mathcal{W}^{b}\left(q^{b}\right)=\left(\partial_{q^{b}} q^{c}\right)^{2} \mathcal{W}^{c}\left(q^{c}\right)+\left(q^{c} ; q^{b}\right)= \\
\left(\partial_{q^{b}} q^{c}\right)^{2}\left[\left(\partial_{q^{c}} q^{a}\right)^{2}\left(q^{a} ; q^{c}\right)\right]+\left(q^{c} ; q^{b}\right)= \\
\left(\partial_{q^{b}} q^{a}\right)^{2} \mathcal{W}^{a}\left(q^{a}\right)+\left(\partial_{q^{b}} q^{c}\right)^{2}\left(q^{a} ; q^{c}\right)+\left(q^{c} ; q^{b}\right),
\end{gathered}
$$

with (14) we obtain the basic relation [2]

$$
\left(q^{a} ; q^{c}\right)=\left(\partial_{q^{c}} q^{b}\right)^{2}\left(q^{a} ; q^{b}\right)-\left(\partial_{q^{c}} q^{b}\right)^{2}\left(q^{c} ; q^{b}\right)
$$

which extends to higher dimensions [3]. This relation, which is a direct consequence of the equivalence principle, actually implies that [2]

$$
\left(q^{a} ; q^{b}\right)=-\frac{\beta^{2}}{4 m}\left\{q^{a}, q^{b}\right\},
$$


where $\beta$ is a dimensional constant and

$$
\{h(x), x\}=\frac{h^{\prime \prime \prime}(x)}{h^{\prime}(x)}-\frac{3}{2}\left(\frac{h^{\prime \prime}(x)}{h^{\prime}(x)}\right)^{2}=\left(\ln h^{\prime}(x)\right)^{\prime \prime}-\frac{1}{2}\left[\left(\ln h^{\prime}(x)\right)^{\prime}\right]^{2},
$$

denotes the Schwarzian derivative.

Since the inhomogeneous term in the transformation of $\mathcal{W}$ must disappear in the classical limit, we have by (17) that the classical phase corresponds to the $\beta \longrightarrow 0$ limit. By Eqs.(13) and (17) it follows that $\mathcal{W}$ itself is a Schwarzian derivative

$$
\mathcal{W}^{v}\left(q^{v}\right)=-\frac{\beta^{2}}{4 m}\left\{q^{0}, q^{v}\right\}
$$

with $q^{0}$ determined by the fact that the $\beta \longrightarrow 0$ limit corresponds to the classical phase. One obtains $[1,2]$

$$
Q=\frac{\beta^{2}}{4 m}\left\{\mathcal{S}_{0}, q\right\}
$$

Eq.(7) and the identity ${ }^{1}$

$$
\left(\partial_{q} \mathcal{S}_{0}\right)^{2}=\frac{\beta^{2}}{2}\left\{e^{\frac{2 i}{\beta} \mathcal{S}_{0}}, q\right\}-\frac{\beta^{2}}{2}\left\{\mathcal{S}_{0}, q\right\},
$$

imply that Eq.(20) is equivalent to

$$
\mathcal{W}=-\frac{\beta^{2}}{4 m}\left\{e^{\frac{2 i}{\beta} S_{0}}, q\right\} .
$$

By $(7)$ and $(20)$ it follows that the equation for $\mathcal{S}_{0}$ we were looking for is $[1,2]$

$$
\frac{1}{2 m}\left(\frac{\partial \mathcal{S}_{0}(q)}{\partial q}\right)^{2}+\mathcal{W}(q)+\frac{\beta^{2}}{4 m}\left\{\mathcal{S}_{0}, q\right\}=0
$$

which is equivalent to (22). It follows that

$$
e^{\frac{2 i}{\beta} S_{0}}=\frac{\psi^{D}}{\psi},
$$

where $\psi^{D}$ and $\psi$ are linearly independent solutions of the stationary Schrödinger equation

$$
\left(-\frac{\beta^{2}}{2 m} \frac{\partial^{2}}{\partial q^{2}}+V(q)\right) \psi=E \psi
$$

Thus, for the "covariantizing parameter" we have

$$
\beta=\hbar,
$$

\footnotetext{
${ }^{1}$ This identity admits a higher dimensional extension [3].
} 
where $\hbar=h / 2 \pi$ and $h$ is the Planck constant.

In Ref.[1] the function $\mathcal{T}_{0}(p)$, defined as the Legendre transform of the reduced action, has been introduced

$$
\mathcal{S}_{0}(q)=p q-\mathcal{T}_{0}(p)
$$

While $\mathcal{S}_{0}(q)$ is the momentum generating function, its Legendre dual $\mathcal{T}_{0}(p)$ is the coordinate generating function

$$
p=\frac{\partial \mathcal{S}_{0}}{\partial q}, \quad q=\frac{\partial \mathcal{T}_{0}}{\partial p} .
$$

The second derivative of $(27)$ with respect to $s=\mathcal{S}_{0}(q)$ yields the "canonical equation"

$$
\left(\partial_{\mathrm{s}}^{2}+\mathcal{U}(\mathrm{s})\right) q \sqrt{p}=0=\left(\partial_{\mathrm{s}}^{2}+\mathcal{U}(\mathrm{s})\right) \sqrt{p}
$$

with the "canonical potential" being

$$
\mathcal{U}(\mathrm{s})=\{q \sqrt{p} / \sqrt{p}, \mathrm{~s}\} / 2=\{q, \mathrm{~s}\} / 2
$$

Observe that the choice of the coordinates $q$ and $q^{v}$, which of course does not imply any loss of generality as both $q$ and $q^{v}$ play the role of independent coordinate in their own system, allows us to look at the reduced action as a scalar function. In particular, since $\mathcal{S}_{0}^{v}\left(q^{v}\right)=\mathcal{S}_{0}(q)$, we see that the transformations (2) leave the Legendre transform of $\mathcal{T}_{0}(27)$ unchanged. Consequently, from $\partial_{q} \mathcal{S}_{0}^{v}\left(q^{v}\right)=\left(\partial_{q} q^{v}\right)^{-1} \partial_{q} \mathcal{S}_{0}(q)$, we have $p \longrightarrow p_{v}=\left(\partial_{q} q^{v}\right)^{-1} p$. However, while the Legendre transform of $\mathcal{T}_{0}$ is, by definition, invariant under the $v$-transformations, this is not the case for the canonical potential $\mathcal{U}$. Nevertheless, there is an important exception as under the $G L(2, \mathrm{C})$ transformations

$$
q^{v}=(A q+B) /(C q+D), \quad p_{v}=\rho^{-1}(C q+D)^{2} p
$$

where $\rho \equiv A D-B C \neq 0$, we have that the Möbius symmetry of the Schwarzian derivative implies

$$
\mathcal{U}(\mathbf{s})=\{(A q+B) /(C q+D), \mathbf{s}\} / 2=\mathcal{U}(\mathbf{s}) .
$$

Therefore we can speak of $G L(2, \mathrm{C})$-symmetry of the canonical equation.

Involutivity of the Legendre transform and the duality

$$
\mathcal{S}_{0} \longleftrightarrow \mathcal{T}_{0}, \quad q \longleftrightarrow p
$$

imply another $G L(2, C)$-symmetry, with the dual version of Eq.(29) being

$$
\left(\partial_{t}^{2}+\mathcal{V}(\mathrm{t})\right) p \sqrt{q}=0=\left(\partial_{t}^{2}+\mathcal{V}(\mathrm{t})\right) \sqrt{q}
$$


where

$$
\mathcal{V}(\mathrm{t})=\{p \sqrt{q} / \sqrt{q}, \mathrm{t}\} / 2=\{p, \mathrm{t}\} / 2
$$

with $t=\mathcal{T}_{0}(p)$. We note that for $p=\gamma / q$ the solutions of (29) and (32) coincide. Therefore we have the self-dual states

$$
\mathcal{S}_{0}=\gamma \ln \gamma_{q} q, \quad \mathcal{T}_{0}=\gamma \ln \gamma_{p} p
$$

where the three constants satisfy $\gamma_{p} \gamma_{q} \gamma=e$. Observe that

$$
\mathcal{S}_{0}+\mathcal{T}_{0}=p q=\gamma, \quad \mathcal{U}(\mathbf{s})=-1 / 4 \gamma^{2}=\mathcal{V}(\mathrm{t})
$$

The canonical equation (29) and its dual (32) correspond to two equivalent descriptions of physical systems that for the self-dual states overlap. Later we will consider another derivation of the self-dual states (34).

Remarkably, the quantum Hamilton-Jacobi equation (23) can be also seen as modification by a "conformal factor" of the CSHJE. In particular, using the identity

$$
\left\{q, \mathcal{S}_{0}\right\}=-\left(\partial_{q} \mathcal{S}_{0}\right)^{-2}\left\{\mathcal{S}_{0}, q\right\}
$$

we have that the canonical potential determines the conformal rescaling $[1,2]$

$$
\frac{1}{2 m}\left(\frac{\partial \mathcal{S}_{0}}{\partial q}\right)^{2}\left[1-\hbar^{2} \mathcal{U}\left(\mathcal{S}_{0}\right)\right]+V(q)-E=0
$$

This shows the basic role of the purely quantum mechanical self-dual state (34) with $\gamma=i \hbar / 2$ as for the $\mathcal{W}^{0}\left(q^{0}\right)$ state, $\mathrm{Eq} .(36)$ has the form

$$
\frac{1}{2 m}\left(\frac{\partial \mathcal{S}_{0}^{0}}{\partial q^{0}}\right)^{2}\left[1-\hbar^{2} \mathcal{U}\left(\mathcal{S}_{0}^{0}\right)\right]=0
$$

whose solution is

$$
\mathcal{S}_{0}^{0}=\frac{i}{2} \hbar \ln \gamma_{q} q^{0}
$$

that is

$$
1-\hbar^{2} \mathcal{U}\left(\frac{i}{2} \hbar \ln \gamma_{q} q^{0}\right)=0
$$

Therefore, in quantum mechanics, the $\mathcal{W}^{0}$ state corresponds to one of the self-dual states (34) labelled by $\gamma$, for which the $\mathcal{S}_{0}$ and $\mathcal{T}_{0}$ descriptions coincide. We note that the zero mode (38) of the conformal factor $1-\hbar^{2} \mathcal{U}$ has been overlooked in literature. 
The solution $\mathcal{S}_{0}^{0}=\frac{i}{2} \hbar \ln \gamma_{q} q^{0}$ solves the problem of finding the trivializing coordinate for which $\mathcal{W}(q) \longrightarrow \mathcal{W}^{0}\left(q^{0}\right)$. Actually, according to (5) we have

$$
q \longrightarrow q^{0}=\gamma_{d}^{-1} e^{\frac{2 i}{\hbar} S_{0}(q)}
$$

We remark that related interesting issues have been recently considered in the [4].

Similarly to the case of general relativity in which the equivalence principle leads to the deformation of the geometry, even in quantum mechanics one should investigate whether the equivalence principle implies a space deformation. In this context, the structure of the quantum Hamilton-Jacobi equation (36) suggests considering an underlying geometrical structure. Actually, Eq.(36) naturally leads to a coordinate transformation depending on the quantum potential. The key point is that (36) can be written in the form

$$
\frac{1}{2 m}\left(\frac{\partial \mathcal{S}_{0}}{\partial \hat{q}}\right)^{2}+V(q)-E=0
$$

where

$$
\left(\frac{\partial q}{\partial \hat{q}}\right)^{2}=\left[1-\hbar^{2} \mathcal{U}\left(\mathcal{S}_{0}\right)\right]
$$

or equivalently (we omit the solution with the minus sign)

$$
d \hat{q}=\frac{d q}{\sqrt{1-\beta^{2}(q)}}
$$

with

$$
\beta^{2}(q)=\hbar^{2} \mathcal{U}\left(\mathcal{S}_{0}\right)=\frac{\hbar^{2}}{2}\left\{q, \mathcal{S}_{0}\right\}
$$

Integrating (42) yields

$$
\hat{q}=\int^{q} \frac{d x}{\sqrt{1-\beta^{2}(x)}} .
$$

We observe that the nature of the coordinate transformation is purely quantum mechanical; in particular

$$
\lim _{h \rightarrow 0} \hat{q}=q .
$$

Equation (45) indicates that in considering the differential structure one should take into account the effect of the potential on space geometry. In this context, the deformation of the classical Hamilton-Jacobi equation amounts to replacing the standard derivative with respect to the classical coordinate $q$ with the derivative with respect to deformed quantum coordinate $\hat{q}$. In other words, the transition from the classical to the quantum regime amounts to a reconsideration of the underlying geometry which is modified by the potential itself. 
A property of the quantum transformation (43) is that it allows to put the quantum Hamilton-Jacobi equation in the classical form. Namely, setting

$$
\begin{aligned}
& \hat{\mathcal{W}}(\hat{q})=\mathcal{W}(q(\hat{q})), \\
& \hat{\mathcal{S}}_{0}(\hat{q})=\mathcal{S}_{0}(q(\hat{q})),
\end{aligned}
$$

it follows that Eq.(36), equivalent to Eq.(37), can be written in the form

$$
\frac{1}{2 m}\left(\frac{\partial \hat{\mathcal{S}}_{0}(\hat{q})}{\partial \hat{q}}\right)^{2}+\hat{\mathcal{W}}(\hat{q})=0
$$

This can be seen as the opposite of the problem, considered by Schiller and Rosen [5], of determining the wave function representation for classical mechanics (see also [6]).

In the standard formulation of the quantum analogue of the Hamilton-Jacobi equation [7], one considers a couple of equations which arise by setting $\psi=R e^{i \mathcal{S}_{0} / \hbar}$, so that for the $\mathcal{W}^{0}\left(q^{0}\right)$ state one chooses $\mathcal{S}_{0}=$ cnst and $R=A q^{0}+B$. Note that setting $\psi=R e^{i \mathcal{S}_{0} / \hbar}$ is suggested by the interpretation of $|\psi|^{2}=R^{2}$ as probability density. On the other hand, it is easy to see that any solution has the form

$$
\psi=\frac{1}{\sqrt{\mathcal{S}_{0}^{\prime}}}\left(A e^{-\frac{i}{\hbar} \mathcal{S}_{0}}+B e^{\frac{i}{\hbar} \mathcal{S}_{0}}\right) .
$$

However, while on the one hand it is not possible to define the Legendre transform of a constant, so that the $\mathcal{S}_{0}-\mathcal{T}_{0}$ duality would be lost, on the other hand, we have that the overlooked solution $\mathcal{S}_{0}^{0}=\frac{i}{2} \hbar \ln \gamma_{q} q^{0}$ for the $\mathcal{W}^{0}$ state still gives the same wave function $\psi=A q^{0}+B$. The form (23) of the quantum Hamilton-Jacobi equation is considered in [8]. In this context we observe that the non-linear relation between $\mathcal{S}_{0}$ and the wave function, which can be also written in the form $\mathcal{S}_{0}(q)=\frac{i}{2} \hbar \ln \left(A \int^{q} \psi^{-2}+B\right) /\left(C \int^{q} \psi^{-2}+D\right)$, is related to a non complete equivalence between the Schrödinger equation and the quantum Hamilton-Jacobi equation (23). Another interesting example of inequivalence between the Schrödinger equation and Eq.(23) is provided in [9] where it has been shown that for bound states the quantum Hamilton-Jacobi equation (23) describes microstates not detected in the Schrödinger representation. This is an interesting example of how the reduced action $\mathcal{S}_{0}$ represents an exhaustive description of quantum mechanics.

The fact that the quantum Hamilton-Jacobi equation admits the classical representation (49) suggests that classically forbidden regions correspond to critical regions for the quantum coordinate. Actually, writing Eq.(45) in the equivalent form $\left(s=\mathcal{S}_{0}(q)\right)$

$$
\hat{q}=\int^{q} d x \frac{\partial_{x} \mathcal{S}_{0}}{\sqrt{-2 m \mathcal{W}}}=\int^{\mathcal{S}_{0}(q)} \frac{d \mathbf{s}}{\sqrt{-2 m \mathcal{W}}},
$$


we see that the integrand is purely imaginary in the classically forbidden regions $\mathcal{W}>0$. Furthermore, since according to (37), for the $\mathcal{W}^{0}$ state the conformal factor vanishes, it follows by (45) that the quantum coordinate for the free particle state with vanishing energy is divergent. To better understand the role of the $\mathcal{W}^{0}$ state it is useful to first rederive the self-dual states (34) by another approach.

The $\mathcal{S}_{0}-\mathcal{T}_{0}$ duality implies that a given physical system may be described either by the $\mathcal{S}_{0}$-picture or by the $\mathcal{T}_{0}$-picture. On general grounds, it is clear that a naturally selected $\mathcal{W}$ state is the one corresponding to the degenerate case in which the $\mathcal{S}_{0}$ and $\mathcal{T}_{0}$ pictures overlap. In order to find this common subspace we consider the interchange of the $\mathcal{S}_{0}$ and $\mathcal{T}_{0}$ pictures given by

$$
q \longrightarrow \tilde{q}=\alpha p \quad p \longrightarrow \tilde{p}=\beta q \text {. }
$$

This implies that

$$
\frac{\partial \tilde{\mathcal{T}}_{0}}{\partial \tilde{p}}=\alpha \frac{\partial \mathcal{S}_{0}}{\partial q}, \quad \frac{\partial \tilde{\mathcal{S}}_{0}}{\partial \tilde{q}}=\beta \frac{\partial \mathcal{T}_{0}}{\partial p}
$$

which is equivalent to

$$
\frac{\partial \tilde{\mathcal{T}}_{0}}{\partial q}=\alpha \beta \frac{\partial \mathcal{S}_{0}}{\partial q}, \quad . \quad \frac{\partial \tilde{\mathcal{S}}_{0}}{\partial p}=\alpha \beta \frac{\partial \mathcal{T}_{0}}{\partial p}
$$

that is

$$
\tilde{\mathcal{S}}_{0}(\tilde{q})=\alpha \beta \mathcal{T}_{0}(p)+\text { cnst }, \quad \tilde{\mathcal{T}}_{0}(\tilde{p})=\alpha \beta \mathcal{S}_{0}(q)+\text { cnst }
$$

Furthermore, since we require that (52) be of order two, we have up to an additive constant

$$
\tilde{\tilde{S}}_{0}=\mathcal{S}_{0}, \quad \tilde{\mathcal{T}}_{0}=\mathcal{T}_{0}
$$

so that

$$
(\alpha \beta)^{2}=1
$$

We observe that $\tilde{\mathcal{S}}_{0}(\tilde{q})$ and $\tilde{\mathcal{T}}_{0}(\tilde{p})$ are basically the Legendre transform of $\mathcal{S}_{0}(q)$ and $\mathcal{T}_{0}(p)$ respectively.

The distinguished $\mathcal{W}$ states are precisely those which are left invariant by (52) and (55), that is

$$
\tilde{\mathcal{S}}_{0}(\tilde{q})=\mathcal{S}_{0}(q)+\text { cnst }
$$

Let us now introduce the Legendre transform of the Hamilton principal function $\mathcal{S}$

$$
\mathcal{S}=p \frac{\partial \mathcal{T}}{\partial p}-\mathcal{T}, \quad \mathcal{T}=q \frac{\partial \mathcal{S}}{\partial q}-\mathcal{S}
$$




$$
p=\frac{\partial \mathcal{S}}{\partial q}, \quad q=\frac{\partial \mathcal{T}}{\partial p} .
$$

Observe that for stationary states

$$
\mathcal{S}(q, t)=\mathcal{S}_{0}(q)-E t, \quad \mathcal{T}(p, t)=\mathcal{T}_{0}(p)+E t
$$

Let us consider the differentials

$$
\begin{gathered}
d \mathcal{S}=\frac{\partial \mathcal{S}}{\partial q} d q+\frac{\partial \mathcal{S}}{\partial t} d t=p d q+\frac{\partial \mathcal{S}}{\partial t} d t \\
d \mathcal{T}=\frac{\partial \mathcal{T}}{\partial p} d p+\frac{\partial \mathcal{T}}{\partial t} d t=q d p+\frac{\partial \mathcal{T}}{\partial t} d t
\end{gathered}
$$

which imply

$$
d \mathcal{S}=d(p q-\mathcal{T})=p d q+q d p-q d p-\frac{\partial \mathcal{T}}{\partial t} d t
$$

that is

$$
\frac{\partial \mathcal{S}}{\partial t}=-\frac{\partial \mathcal{T}}{\partial t}
$$

This equation connects the $\mathcal{S}$ and $\mathcal{T}$ pictures through the time evolution. By (55) (57) (58) and (61) we have that the distinguished states correspond to

$$
\mathcal{S}= \pm \mathcal{T}+\text { cnst }
$$

As (66) should be stable under time evolution, the relation (65) fixes the sign ambiguity and sets

$$
\alpha \beta=-1
$$

Therefore, the distinguished $\mathcal{W}$ states correspond to

$$
\mathcal{S}=-\mathcal{T}+\text { cnst }
$$

Since $\mathcal{S}=p q-\mathcal{T}$, we have

$$
p q=\gamma,
$$

where $\gamma$ is a constant. Therefore, the distinguished states are precisely the self-dual states $(34)$.

We have seen that the self-dual state with $\gamma=i \hbar / 2$ corresponds to the $\mathcal{W}^{0}$ state. The fact that it corresponds to the distinguished state connecting the $\mathcal{S}_{0}$ and $\mathcal{T}_{0}$ pictures, indicates that this state corresponds to a critical point for the coordinate transformation. In this context the observed divergence for $\hat{q}$ corresponding to the $\mathcal{W}^{0}$ state is not a surprise. A 
property of this state is that it corresponds to $\frac{1}{2 m}\left(\partial_{q^{0}} \mathcal{S}_{0}^{0}\right)^{2}=-\hbar^{2} /\left(8 m q^{0^{2}}\right)$ showing that we cannot consider the momentum as in classical mechanics. Furthermore, more generally, we can see how the $G L(2, \mathrm{C})$-symmetry makes $\frac{1}{2 m}\left(\partial_{q} \mathcal{S}_{0}\right)^{2}$ undetermined. Namely, due to the Möbius symmetry of the Schwarzian derivative, one has

$$
\mathcal{W}=-\frac{\hbar^{2}}{4 m}\left\{e^{\frac{2 i}{\hbar} S_{0}}, q\right\}=-\frac{\hbar^{2}}{4 m}\left\{\frac{A e^{\frac{2 i}{\hbar} S_{0}}+B}{C e^{\frac{2 i}{\hbar} S_{0}}+D}, q\right\} .
$$

It follows by (21) that what is invariant is not $\left(\partial_{q} \mathcal{S}_{0}\right)^{2}$ but rather $\left(\partial_{q} \mathcal{S}_{0}\right)^{2}+\hbar^{2}\left\{\mathcal{S}_{0}, q\right\} / 2$. This indicates that the "kinetic term" $\frac{1}{2 m}\left(\partial_{q} \mathcal{S}_{0}\right)^{2}$ and the quantum correction $Q=\frac{\hbar^{2}}{4 m}\left\{\mathcal{S}_{0}, q\right\}$ make sense separately in the classical regime only where $Q \longrightarrow 0$. The role of the quantum correction $Q$ is somehow reminiscent of the relativistic rest energy, as it is an intrinsic property of the particle.

We now show that the quantum Hamilton-Jacobi equation has an explicit geometrical formulation. In order to do this we use a result by Flanders [10] who showed that the Schwarzian derivative can be interpreted as curvature of an equivalence problem for curves in $\mathbf{P}^{1}$. One first introduces a frame for $\mathrm{P}^{1}$ consisting of a pair $\mathrm{x}, \mathrm{y}$ of point in affine space $\mathbf{A}^{2}$ such that $[\mathbf{x}, \mathbf{y}]=1$, where $[\mathbf{x}, \mathbf{y}]=x_{1} y_{2}-x_{2} y_{1}$ is the area function. Considering the moving frame $s \longrightarrow\{\mathbf{x}(s), \mathbf{y}(s)\}$ and differentiating $[\mathbf{x}, \mathbf{y}]=1$ yields the structure equations

$$
\mathbf{x}^{\prime}=a \mathbf{x}+b \mathbf{y}, \quad \mathbf{y}^{\prime}=c \mathbf{x}-a \mathbf{y}
$$

Given a map $\phi=\phi(s)$ from a domain to $\mathbf{P}^{1}$ one can choose a moving frame $\mathbf{x}(s), \mathbf{y}(s)$ in such way that $\phi(s)$ is represented by $\mathbf{x}(s)$. Observe that this map can be seen as a curve in $\mathbf{P}^{\mathbf{1}}$. Two mappings $\phi$ and $\psi$ are said equivalent if $\psi=\pi \circ \phi$ with $\pi$ a projective transformation on $\mathbf{P}^{1}$. One easily see that if $b=0$ then $\phi$ is constant. If $b$ is never vanishing there are two possible cases with the associated natural moving frames being [10]

$$
\mathrm{x}^{\prime}=\mathrm{y}, \quad \mathrm{y}^{\prime}=-k \mathrm{x}
$$

and

$$
\mathbf{x}^{\prime}=-\mathbf{y}, \quad \mathbf{y}^{\prime}=k \mathbf{x} .
$$

It turns out that $\mathbf{x}, \mathbf{y}$ are determined up to a sign and $k$ is an invariant. That is if $\mathbf{x}_{1}^{\prime}=\mathbf{y}_{1}$, $\mathbf{y}_{1}^{\prime}=-k_{1} \mathbf{x}_{1}$, then $\mathbf{x}_{1}= \pm \mathbf{x}, \mathbf{y}_{1}= \pm \mathbf{y}$ and $k_{1}=k$.

If $s \longrightarrow \mathbf{z}(s)$ is an affine representative of $\phi$ then $\mathbf{z}=\lambda \mathbf{x}$ where $\lambda(s)$ is never vanishing. It is easy to see that

$$
2 k=\frac{\left[\mathbf{z}, \mathbf{z}^{\prime \prime \prime}\right]+3\left[\mathbf{z}^{\prime}, \mathbf{z}^{\prime \prime}\right]}{\left[\mathbf{z}, \mathbf{z}^{\prime}\right]}-\frac{3}{2}\left(\frac{\left[\mathbf{z}, \mathbf{z}^{\prime \prime}\right]}{\left[\mathbf{z}, \mathbf{z}^{\prime}\right]}\right)^{2}
$$


Given a function $z(s)$, this can be seen as the non-homogeneous coordinate of a point in $\mathbf{P}^{1}$. Therefore, we can associate to $z$ the map $\phi$ defined by $s \longrightarrow(1, z(s))=\mathbf{z}(s)$, so that $\left[\mathbf{z}, \mathbf{z}^{\prime}\right]=z^{\prime},\left[\mathbf{z}, \mathbf{z}^{\prime \prime}\right]=z^{\prime \prime},\left[\mathbf{z}, \mathbf{z}^{\prime \prime \prime}\right]=z^{\prime \prime \prime},\left[\mathbf{z}^{\prime}, \mathbf{z}^{\prime \prime}\right]=0$ and the curvature becomes $[10]$

$$
k=\frac{1}{2}\{z, s\} .
$$

Therefore, we have

$$
\mathcal{W}=-\frac{\hbar^{2}}{4 m}\left\{e^{\frac{2 i}{\hbar} s_{0}}, q\right\}=-\frac{\hbar^{2}}{2 m} k_{\mathcal{W}}
$$

and

$$
Q=\frac{\hbar^{2}}{4 m}\left\{\mathcal{S}_{0}, q\right\}=\frac{\hbar^{2}}{2 m} k_{Q},
$$

where $k_{\mathcal{W}}$ is the curvature associated to the map

$$
q \longrightarrow\left(1, e^{\frac{2 i}{n} \mathcal{S}_{0}(q)}\right),
$$

whereas the curvature $k_{Q}$ is associated to the map

$$
q \longrightarrow\left(1, \mathcal{S}_{0}(q)\right) .
$$

Remarkably, the function defining the map (78) coincides with the trivializing map (5).

The Schrödinger equation takes the geometrical form

$$
\left(\frac{\partial^{2}}{\partial q^{2}}+k_{\mathcal{W}}\right) \psi=0
$$

Furthermore, the identity (21) can be now seen as difference of curvatures

$$
\left(\partial_{q} \mathcal{S}_{0}\right)^{2}=\hbar^{2} k_{\mathcal{W}}-\hbar^{2} k_{Q},
$$

and the quantum Hamilton-Jacobi equation (23) can be written in the form

$$
\frac{1}{2 m}\left(\frac{\partial \mathcal{S}_{0}(q)}{\partial q}\right)^{2}+\mathcal{W}(q)+\frac{\hbar^{2}}{2 m} k_{Q}=0 .
$$

As we noticed above, with respect to the standard solution for the free quantum state of vanishing energy, $\mathcal{S}_{0}^{0}=$ cnst, which is the same for classical mechanics, one has that what is vanishing is not $\partial_{q^{0}} \mathcal{S}_{0}$ but $\frac{1}{2 m}\left(\frac{\partial \mathcal{S}_{0}^{0}\left(q^{0}\right)}{\partial q^{0}}\right)^{2}+\frac{\hbar^{2}}{4 m}\left\{\mathcal{S}_{0}^{0}, q^{0}\right\}=0$, so that one has $\mathcal{S}_{0}^{0}=\frac{i}{2} \hbar \ln \gamma_{q} q^{0}$ and we have a non zero curvature term associated to the free particle with vanishing energy.

We observe that the equivalence principle implies the quantum Hamilton-Jacobi equation also in higher dimensions [3]. In deriving it one uses the higher dimensional generalization of (23). This suggests a higher dimensional generalization of Flanders result. 
We observe that our investigation is related to the approach in [11], further developed by Carroll in [12], where it has been shown that the space coordinate is proportional to the Legendre transform of the prepotential $\mathcal{F}$, defined by $\psi^{D}=\partial_{\psi} \mathcal{F}$, with respect to the square of the wave function.

Finally, we note that very recently related issues have been considered in [13].

It is a pleasure to thank G. Bertoldi, G. Bonelli and M. Tonin for interesting discussions. Work supported in part by DOE Grant No. DE-FG-0586ER40272 (AEF) and by the European Commission TMR programme ERBFMRX-CT96-0045 (MM).

\section{References}

[1] A.E. Faraggi and M. Matone, hep-th/9705108.

[2] A.E. Faraggi and M. Matone, hep-th/9711028.

[3] G. Bertoldi, A.E. Faraggi and M. Matone, paper in preparation.

[4] V. Periwal, hep-th/9709200.

[5] R. Schiller, Phys. Rev. 125 (1962) 1100; ibidem 1109.

A. Rosen, Am. J. Phys. 32 (1964) 597; Found. Phys. 16 (1986) 687.

[6] P.R. Holland, The Quantum Theory of Motion, Cambridge Univ. Press (Cambridge 1993).

[7] L.D. Landau and E.M. Lifschitz, Quantum Mechanics, Pergamon Press (Oxford 1958).

[8] A. Messiah, Quantum Mechanics, Vol.1, North-Holland (Amsterdam 1995).

[9] E. Floyd, quant-ph/9707051.

[10] H. Flanders, J. Diff. Geom. 4 (1970) 515.

[11] A.E. Faraggi and M. Matone, Phys. Rev. Lett. 78 (1997) 163.

[12] R. Carroll, hep-th/9607219; hep-th/97010216; hep-th/9702138; Nucl. Phys. B502 (1997) 561.

[13] J.S. Anandan, gr-qc/9712015. 